\title{
Physician and patient use of and attitudes toward complementary and alternative medicine in the treatment of infertility
}

\author{
Natalie A. Clark*, Matthew A. Will, Molly B. Moravek, Xiao Xu, Senait Fisseha \\ Department of Obstetrics and Gynecology, Division of Reproductive Endocrinology and Infertility, University of Michigan, Ann Arbor, USA
}

\section{A R T I C L E I N F O}

\section{Article history:}

Received 10 December 2012

Received in revised form 16 March 2013

Accepted 28 May 2013

\section{Keywords:}

Complementary and alternative medicine

Infertility

Knowledge

Patients

Perceptions

Physicians

\begin{abstract}
A B S T R A C T
Objective: To determine use of and attitudes toward complementary and alternative medicine (CAM) among infertility patients and subspecialty physicians. Methods: Infertility patients were asked to complete anonymous written surveys at an academic infertility practice; members of the Society for Reproductive Endocrinology and Infertility were electronically surveyed. Both groups were assessed regarding their use of and attitudes toward CAM. Results: The response rate was 32.1\% (115/358) among patients and 22.6\% (225/995) among physicians $(P<0.05)$. In total, 105 (91.3\%; 95\% confidence interval $[\mathrm{CI}], 85.8-96.2)$ patients used CAM, and 84 (73.0\%; 95\% CI, 64.9-81.1) regarded it as beneficial to their fertility treatment. However, only 30 (26.1\%; $95 \%$ CI, 18.0-34.0) patients reported CAM use to physicians, with the most common reason being that they were “never asked." Overall, 202 (89.8\%; 95\% CI, 85.9-93.8) physicians reported inquiring about CAM. Conclusion: Significant discrepancies exist between subfertile patients and physicians in attitudes toward the use of CAM. The current prevalence of CAM use among infertility patients requires greater physician attention and justifies further study on the risks and benefits of integrating CAM into the biomedical treatment of infertility.
\end{abstract}

Published by Elsevier Ireland Ltd. on behalf of International Federation of Gynecology and Obstetrics.

\section{Introduction}

Complementary and alternative medicine (CAM) is a growing trend in contemporary medicine [1]. It is defined by the National Center for Complementary and Alternative Medicine as "a group of diverse medical and health care systems, practices and products that are not presently considered to be part of conventional medicine" [2]. Complementary and alternative medicine therapies include a wide range of practices, such as the use of acupuncture and herbal supplements, and are mediated through a variety of measurable and immeasurable mechanisms. For example, acupuncture works along the body's meridians, stimulating $q i$ [3], while herbal medicines can contain a variety of bioactive substances [4].

Over the past 3 decades, the use of CAM by both patients and physicians has increased markedly, as has the use of CAM for fertility treatment $[1,5]$. The National Institutes of Health National Center for Complementary and Alternative Medicine reports that $38 \%$ of adults use some form of CAM [6]. A recent study demonstrated that $29 \%$ of couples seeking infertility care had used CAM for treatment of infertility [7]. Similarly, a Canadian observational study demonstrated a $30 \%$ prevalence of CAM use among men presenting for infertility

\footnotetext{
* Corresponding author at: Department of Obstetrics and Gynecology, L4100 Women's Hospital, SPC 5276, University of Michigan Medical Center, 1500 East Medical Center Drive, Ann Arbor, MI 48109-0276, USA. Tel.: + 1734474 4035; fax: + 17349368745.

E-mail address: naclark@med.umich.edu (N.A. Clark).
}

evaluation [8]. Subsequently, an integrated clinical structure is emerging where biomedical treatment and CAM are blended within the fertility practice [1].

Infertile couples seeking to conceive may try CAM before they commence treatment or they may use it as an adjunct to infertility care. An Australian study found that patients turn to CAM to maximize their success with assisted reproductive technology (ART), to seek a more personalized treatment, to maintain wellbeing while undergoing ART, or because they are desperate for a positive result [9]. While CAM may, theoretically, provide a therapeutic benefit, highquality randomized controlled trials assessing the risks and benefits of CAM are lacking, particularly with regard to infertility $[6,10]$. Regardless of purported benefits, given the prevalence of CAM in contemporary infertility practice, it is essential to assess the potential role of CAM in the treatment of infertility and to assess the depth and breadth of its use. The aim of the present survey study was to determine the use of and attitudes toward CAM among infertility patients and physicians.

\section{Materials and methods}

The study consisted of 2 arms. The first assessed patient use of and attitudes toward CAM; the second assessed physician use of and attitudes toward CAM. In the patient arm, consecutively seen patients presenting for infertility care at the University of Michigan, Ann Arbor, MI, USA, were asked to complete an anonymous written survey between August 1 and October 31, 2010. In the physician 
arm, anonymous surveys were electronically sent to members of the Society for Reproductive Endocrinology and Infertility (SREI). The survey results were accumulated between March 1 and April 15, 2011. Patient and physician surveys included a cover letter detailing the nature of the study. Because all data were de-identified upon receipt and because the content of the survey was not sensitive in nature, the study received exempt designation from the University of Michigan Institutional Review Board.

Both groups were assessed on the use of and general attitudes toward CAM. General attitudes were measured using a 5-point Likert scale ( $1=$ strongly agree; $5=$ strongly disagree). Response percentages were analyzed and 95\% confidence intervals (CIs) were calculated. Differences in response rates were calculated using a 2-tailed test for proportions. SPSS (IBM, Armonk, NY, USA) was used for statistical analysis. $P<0.05$ was considered to be statistically significant.

\section{Results}

Of the 358 patients who met the inclusion criteria, 115 (32.1\%) agreed to participate and were included in the present analysis; of the 995 physicians surveyed, $225(22.6 \%)$ responded $(P<0.05)$. Provider demographics are provided in Table 1 . Patient demographics were not collected to ensure anonymity.

Complementary and alternative medicine use was reported by a majority of patients surveyed (105 [91.3\%]; 95\% CI, 85.8-96.2) (Fig. 1). Eighty-four (73.0\%; 95\% Cl, 64.9-81.1) patients perceived CAM to be beneficial to their infertility treatment (Fig. 2). However, only 30 (26.1\%; 95\% CI, 18.0-34.0) patients reported CAM use to their physicians, with the most common reason being that they were "never asked."

In total, 202 (89.8\%; 95\% CI, 85.9-93.8) SREI physicians reported that they inquired about CAM use among their patients (Fig. 3). In fact, large proportions of physicians reported referring patients for acupuncture (136 [60.4\%]; 95\% CI, 54.8-66.1) and massage (92 [40.9\%]; 95\% CI, 35.2-46.5). Additionally, while not actively referring or providing such services, many physicians reported endorsing the use of nutritional supplements (92 [40.9\%]; 95\% CI, 35.2-46.5), prayer or spiritual healing (106 [47.1\%]; 95\% CI, 41.4-52.9), meditation (123 [54.7\%];

Table 1

Provider characteristics $(\mathrm{n}=225)^{\mathrm{a}}$

\begin{tabular}{ll}
\hline Characteristic & Value \\
\hline Age, y & $49.03 \pm 9.83$ \\
Gender & $118(52.4)$ \\
Male & $106(47.1)$ \\
Female & \\
Degree & $214(95.1)$ \\
MD & $2(0.9)$ \\
DO & $4(1.8)$ \\
Other & \\
Specialty & $219(97.3)$ \\
Reproductive endocrinology and infertility & $0(0.0)$ \\
Urology (male infertility) & $1(0.4)$ \\
Other (andrology/embryology) & \\
Race & $184(81.8)$ \\
White & $4(1.8)$ \\
African American & $25(11.1)$ \\
Asian & $1(0.4)$ \\
Hispanic & $6(2.7)$ \\
Multiracial & $1(0.4)$ \\
Other & \\
Ask about CAM & $202 / 221(91.4 ; 95 \% \mathrm{CI}, 88.1-94.7)$ \\
Yes & $19 / 221(8.6 ; 95 \% \mathrm{CI}, 5.3-11.8)$ \\
No & \\
\hline
\end{tabular}

Abbreviation: $\mathrm{CI}$, confidence interval.

a Values are given as mean \pm SD or number (percentage) unless otherwise indicated.
95\% CI, 48.9-60.4), psychotherapy (104 [46.2\%]; 95\% CI, 40.5-51.9), and movement/physical therapy (116 [51.6\%]; 95\% CI, 45.8-57.3).

Most physicians did not strongly believe in the effectiveness of CAM therapies, and only a small proportion of physicians found any CAM therapy to be effective. Physicians believed the following therapies to be moderately effective: acupuncture (115 [51.1\%]; $95 \% \mathrm{CI}$, 45.4-56.9); biofeedback (82 [36.4\%]; 95\% CI, 30.9-42.0); chiropractic manipulation (64 [28.4\%]; 95\% CI, 23.3-33.6); hypnosis (65 [28.9\%]; 95\% CI, 23.7-34.1); massage (83 [36.9\%]; 95\% CI, 33.1-42.3); prayer/ spiritual healing (80 [35.6\%]; 95\% CI, 30.1-41.1); meditation (105 [46.7\%]; 95\% CI, 40.9-52.4); and psychotherapy (108 [48.0\%]; 95\% CI, 42.3-53.8). Although a large number of providers reported that manipulative and body-based practices were moderately effective, 110 (48.9\%; 95\% CI, 43.1-56.6) reported therapeutic or healing touch to be potentially harmful to patients.

Despite concerns regarding lack of effectiveness and potential harm related to CAM, 169 (75.1\%; 95\% CI, 70.1-80.3) physicians agreed or strongly agreed that clinical care should integrate the best conventional and CAM practices. Similarly, most physicians agreed or strongly agreed that CAM includes ideas and methods from which conventional medicine could benefit (167 [4.2\%]; 95\% CI, 69.6-79.6) and that CAM holds promise for the treatment of symptoms, conditions, and diseases (156 [69.3\%]; 95\% CI, 64.3-75.2). Furthermore, 178 (79.1\%; 95\% CI, 74.6-84.1) physicians agreed or strongly agreed that health professionals should be able to advise their patients about commonly used CAM methods. Overall, 150 (66.7\%; 95\% CI, 61.6-72.4) providers disagreed that CAM is a threat to public health, and $131(58.2 \% ; 95 \% \mathrm{CI}$, 52.6-64.0) agreed that knowledge about CAM is as important to themselves as providers as it is to their patients.

\section{Discussion}

The present study demonstrates that a large majority of patients are using CAM in addition to conventional infertility treatment; however, they feel uncomfortable disclosing their CAM use to physicians. This is further complicated by the fact that, while many physicians are uncertain of the efficacy of CAM, they are open to-and often support-the use of these therapies and agree that clinical care should integrate the best conventional and CAM practices.

The study confirms prior observations of other investigators $[1,5,7,9]$ that CAM has become an inextricable part of modern infertility practice and that, by remaining open and educating themselves, providers can help to create a blended model that meets a breadth of patient needs. Given the large number of patients reporting CAM use, there is a fundamental need for more clinical research to identify whether there is significant evidence for or against any CAM therapies, either individually or in combination. As scientific evidence supporting the efficacy of CAM increases [1,5], those therapies that are proven to be effective need to be fully considered by practitioners and incorporated into the clinical armamentarium of contemporary infertility practice. Additionally, the discrepancy in attitudes seen in the present study demonstrates an increasing need to address this topic with infertility patients-whether simply inquiring about possible use or holding more in-depth conversations about risks and benefits.

The present study was not without limitations. Primarily, demographic information was not collected for the patient population in order to maintain anonymity. Beliefs held about CAM may vary by ethnicity; for example, Chinese patients may be more likely to use Chinese herbal medicines and acupuncture. It is possible that the present results were biased such that patients who were interested in CAM preferentially responded. However, given the substantial number of both patient and physician responders, this potential bias may be tempered. Furthermore, it is possible that, in a moderately sized academic reproductive endocrinology and infertility practice, patients would decline to answer surveys given concerns for anonymity. Members of the SREI were surveyed electronically via email; this format 


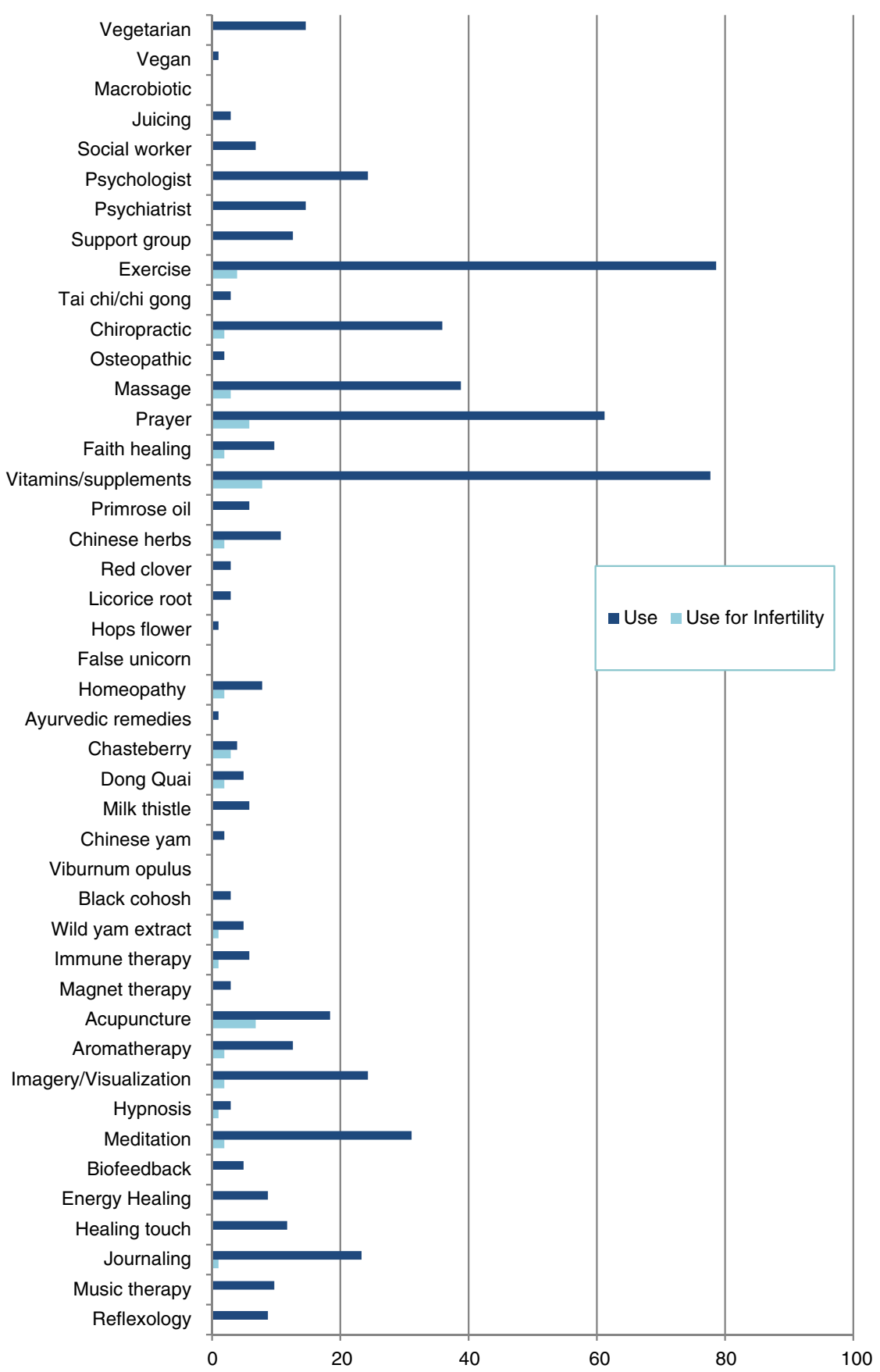

Fig. 1. Use of complementary and alternative medicine reported by patients. Values are shown as percentages.

was chosen based on ease of distribution and the rapid nature by which data could subsequently be collected and analyzed. While this involved a maintained database, it is possible that a number of e-mail addresses were void or incorrect, thus producing a lower response rate than potentially justified. Additionally, no incentives were used, which have been shown to be effective at improving response rates in survey research. The academic literature has documented that low response rates to physician surveys are common [11], particularly for surveys distributed in an electronic format [12]. Additionally, and potentially clouding the answers, the survey asked providers about effectiveness in general regarding each of the therapies, but not effectiveness in what arena. For example, substantial research indicates that many CAM therapies mediate depression and anxiety in the infertile population, without necessarily affecting clinical pregnancy or live birth rates [13]. These issues might be better addressed in future, more specific studies.

As data regarding the biomedical effectiveness of CAM therapies lag behind patients' use of CAM, practitioners caring for infertile patients will increasingly be called upon to individually assess the safety and efficacy of these modalities. Undeniably, personal biases have the potential to color clinical counseling, and it is clear that many providers find CAM to be minimally effective. Regardless, CAM use is widespread among infertility patients and is largely underreported, potentially owing to discomfort with disclosure. Since an abundance of practitioners reported inquiring about CAM use, it is unlikely that providers are not asking but perhaps they are not asking in the right way. Continued investigation into the fundamental basis and biological mechanisms of CAM is essential to evaluate the use of routine 


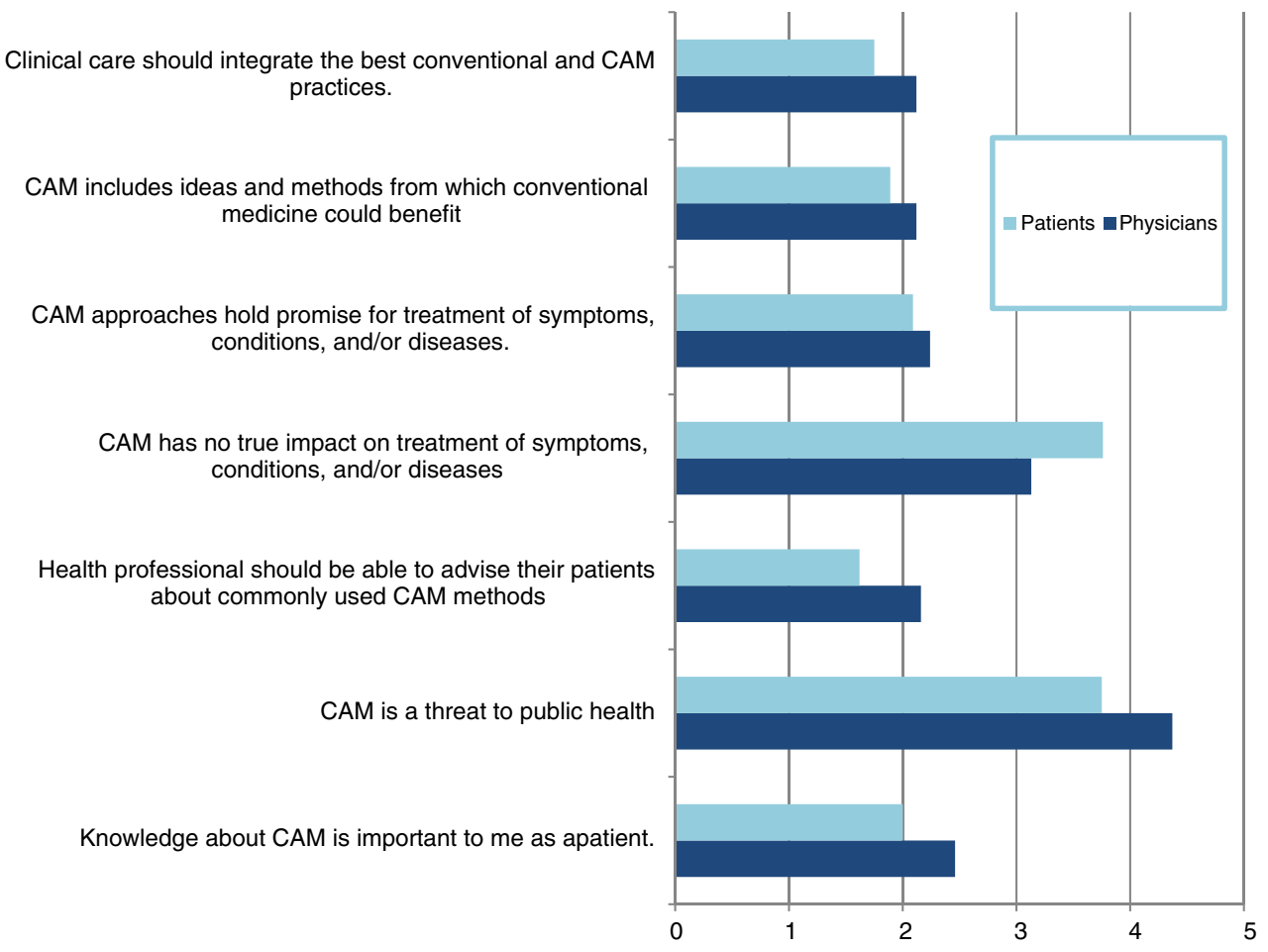

Fig. 2. General attitude assessment regarding complementary and alternative medicine. Measured using a 5-point Likert scale ( $1=$ strongly agree; $5=$ strongly disagree).

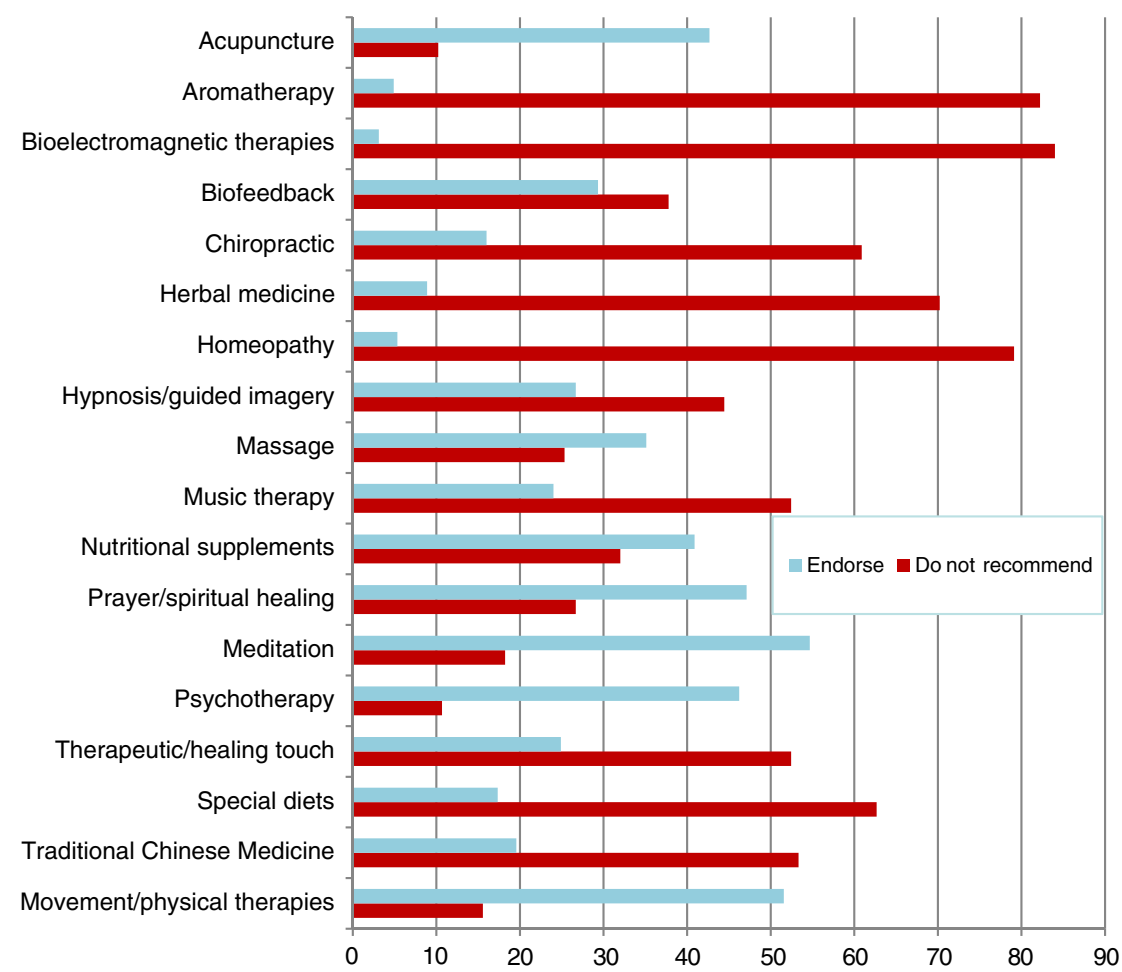

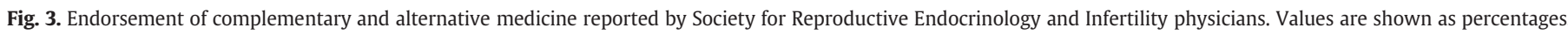

clinical recommendation; however, perhaps by examining individual perspectives and biases, physicians will be better able to provide comprehensive clinical counseling.

\section{Conflict of interest}

The authors have no conflicts of interest.

\section{References}

[1] Pan SY, Gao SH, Zhou SF, Tang MK, Yu ZL, Ko KM. New perspectives on complementary and alternative medicine: an overview and alternative therapy. Altern The Health Med 2012;18(4):20-36.

[2] National Center for Complementary and Alternative Medicine. What Is Complementary and Alternative Medicine? http://nccam.nih.gov/health/whatiscam. Published October, 2008. Updated May, 2012. Accessed November 28, 2012.

[3] NIH Consensus Conference. Acupuncture. JAMA 1998;280(17):1518-24. 
[4] Bent S, Ko R. Commonly used herbal medicines in the United States: a review. Am J Med 2004;116(7):478-85.

[5] Upchurch DM, Chyu L, Greendale GA, Utts J, Bair YA, Zhang G, et al. Complementary and alternative medicine use among American women: findings from The National Health Interview Survey, 2002. J Womens Health (Larchmt) 2007;16(1):102-13.

[6] Barnes J, Abbot NC, Harkness EF, Ernst E. Articles on complementary medicine in the mainstream medical literature: an investigation of MEDLINE, 1966 through 1996. Arch Intern Med 1999;159(15):1721-5.

[7] Smith JF, Eisenberg ML, Millstein SG, Nachtigall RD, Shindel AW, Wing H, et al. The use of complementary and alternative fertility treatment in couples seeking fertility care: data from a prospective cohort in the United States. Fertil Steril 2010;93(7): 2169-74.

[8] Zini A, Fischer MA, Nam RK, Jarvi K. Use of alternative and hormonal therapies in male infertility. Urology 2004;63(1):141-3.
[9] Rayner JA, McLachlan HL, Forster DA, Cramer R. Australian women's use of complementary and alternative medicines to enhance fertility: exploring the experiences of women and practitioners. BMC Complement Altern Med 2009;9:52.

[10] Bennington LK. Can complementary/alternative medicine be used to treat infertility? MCN Am J Matern Child Nurs 2010;35(3):140-7.

[11] Thorpe C, Ryan B, McLean SL, Burt A, Stewart M, Brown JB, et al. How to obtain excellent response rates when surveying physicians. Fam Pract 2009;26(1):65-8.

[12] Shih TH, Fan X. Comparing response rates in e-mail and paper surveys: A metaanalysis. Educ Res Rev 2009;4(1):26-40.

[13] Cousineau TM, Domar AD. Psychological impact of infertility. Best Pract Res Clin Obstet Gynaecol 2007;21(2):293-308. 